\title{
Joint Attention Profiles for Children with Autism in Interactions with Augmentative and Alternative Communication Systems
}

\author{
John W McCarthy', Jamie A Broach², Joann P Benigno \\ 'Ohio University, Athens, United States; ${ }^{2}$ Ohio Valley Educational Service Center, Cambridge, United States
}

Seven preschool children ( $M$ age $=3 ; 11$; range $=2 ; 10-5 ; 7$ ) with autism or related pragmatic difficulties participated in two structured interactions with an AAC system. In the aligned interaction, the system was placed at the chest level of the experimenter with the actual book to the side. In the divided interaction, the system was placed to one side of the experimenter and the book was placed to the other side. Based on states of joint attention (JA) engagement during the AAC interactions, two subgroups emerged: beginning coordinators and active coordinators. JA engagement states with the caregiver, performance on the Early Social Communication Scales, and caregiver report of language and communication skills supported the observations during the AAC interactions. Future research directions and implications for therapy are discussed.

Keywords: Autism, Joint attention, Augmentative and alternative communication

\section{INTRODUCTION}

Joint attention (JA) is a key intervention component in addressing the needs of children with Autism Spectrum Disorders (ASD) with limited functional speech [1]. Because social communication difficulties are a major feature of ASD, early intervention is crucial [2]. Designing intervention protocols to support advances in joint attention is important since JA is related to expressive and receptive language skills $[3,4]$. Further, there is a need for guidance on managing the attentional demands of social communication interactions for children with ASD, particularly when natural speech does not adequately meet their daily communication needs $[5,6]$.

One strategy used to enhance communication in children with ASD is Augmentative and Alternative Communication (AAC) which includes communication modalities that do not require anything but the individual's body (e.g. signing or gesturing) as well as strategies involving external equipment such as pictures or computer based systems with synthesized voice output [7]. Adopting AAC early on, particularly for children with autism who are nonspeaking, may positively impact their subsequent speech, language, and social communication skills [8]. Shifting attention between an external communication aid and their communication partner may be more challenging for children with autism [9]. It follows, then, that creating a situation that is easiest for the child to attend to the communication partner, the system, and object(s) of interest will

(C) 2016 The Korean Association of SpeechLanguage Pathologists

This is an Open Access article distributed under the terms of the Creative Commons Attribution Non-Commercial License (http:// creativecommons.org/licenses/by-nc/4.0/) which permits unrestricted non-commercial use, distribution, and reproduction in any medium, provided the original work is properly cited. 
generate the most beneficial outcomes.

Previous investigations have examined the nature of joint attention interactions shared between children using sign language, their adult communication partners, and objects [10]. Findings suggest that the complexity of the interaction hindered children's abilities to process referential language because children had to shift their focus away from the objects to attend to what the adult was signing [11]. Although akin, the aforementioned scenario would be even more complex with the introduction of a computerized system into the interaction [9].

In an attempt to simulate the complexity of joint exchanges involving AAC systems, Smith et al [12]. examined how the placement of an aided AAC system impacted the shifts of attention in a group of sixteen 9 to 14 month-old beginning communicators without disabilities. The interactions involved the infant, the experimenter, an aided AAC system, and the physical book and 2 conditions: aligned (the system was directly in front of the experimenter/aligned with the experimenter's eye gaze) and divided (the system was off to the side/divided from the experimenter's eye gaze). Infants engaged in more frequent episodes of coordinated JA with both an adult and an AAC system when the system was in an aligned position than in the divided position. A few of the infants exhibited attention shifting between the AAC system and a physical book, as opposed to shifting their attention between the system and the experimenter. This was discussed in a subsequent publication as aided symbol-infused joint engagement [9].

\section{The present study}

The goal of this study was to describe JA patterns of children with autism participating in a computerized literacy activity involving an experimenter and an AAC system. The core research question was: What patterns of joint attention engagement episodes emerged in the context of literacy activities with AAC systems?

\section{METHODS}

\section{Participants}

Seven children ( 2 females; 5 males) participated. This sample size is appropriate for a preliminary investigation. Children ranged from $2 ; 10$ years to $5 ; 7$ years $(M=3 ; 11, S D=0 ; 10)$, all had a diagnosis of ASD or related pragmatic difficulties, and all were receiving speech and language services at the university clinic hosting the summer class (see below). See Table 1 for a summary of demographic information and caregiver report of language comprehension and expression by participant. Inclusionary criteria for participation were: 1) comprehension of symbols that allowed them to make choices between two objects at minimum and 2) the ability to direct-select an item via finger pointing as reported by parents and as observed during the Early Social Communication Scales

Table 1. Demographic Information of the Seven Participating Children

\begin{tabular}{|c|c|c|c|c|c|c|c|}
\hline \multirow[b]{2}{*}{ Participant } & \multirow[b]{2}{*}{ Age } & \multirow[b]{2}{*}{ Sex } & \multicolumn{2}{|c|}{ CSBS Questionnaire } & \multirow[b]{2}{*}{ Diagnosis } & \multirow[b]{2}{*}{ Mode of communication } & \multirow[b]{2}{*}{ School placement } \\
\hline & & & $\begin{array}{l}\text { \# of words } \\
\text { expressed }\end{array}$ & $\begin{array}{c}\text { Comprehension } \\
\text { level }\end{array}$ & & & \\
\hline 1 & $5 ; 7$ & M & $<5$ & Words & Autism & $\begin{array}{l}\text { Gestures, beginning requesting } \\
\text { with picture cards }\end{array}$ & Integrated classroom \\
\hline 2 & $4 ; 3$ & M & $>15$ & Sentences & Autism & $\begin{array}{l}\text { Gestures, verbal } \\
\text { approximations }\end{array}$ & $\begin{array}{l}\text { Integrated preschool } \\
\text { classroom with } \\
\text { intervention specialist }\end{array}$ \\
\hline 3 & $3 ; 4$ & M & $<5$ & Words & PDD-NOS & $\begin{array}{l}\text { Gestures, sounds, pointing, } \\
\text { PECS }\end{array}$ & Integrated classroom \\
\hline 4 & $4 ; 1$ & M & $5-15$ & Sentences & Autism & $\begin{array}{l}\text { Gestures, manipulation of } \\
\text { partner }\end{array}$ & $\begin{array}{l}\text { Special education; Daily } \\
\text { contact with peers } \\
\text { without disabilities }\end{array}$ \\
\hline 5 & $3 ; 2$ & M & $5-15$ & Sentences & $\begin{array}{l}\text { Developmental Lang. } \\
\text { Delay }\end{array}$ & Not reported & $\begin{array}{l}\text { Integrated preschool } \\
\text { classroom }\end{array}$ \\
\hline 6 & $4 ; 7$ & $\mathrm{~F}$ & $>15$ & Sentences & $\begin{array}{l}\text { Sensory Integration and } \\
\text { Neurological deficits }\end{array}$ & Gestures, words & Integrated preschool \\
\hline 7 & $2 ; 10$ & $\mathrm{~F}$ & $<5$ & Words & Autism & Gestures, sounds & Not in school \\
\hline
\end{tabular}


(ESCS) testing. Participants were recruited from a university clinic summer class for children with social communication difficulties after obtaining IRB approval.

\section{Procedures}

All participants were administered the Early Social Communication Scales and six participated in a free play interaction with a primary caregiver for 10 minutes [13]. Caregivers completed a demographic questionnaire and the Communication and Symbolic Behavior Scale: Developmental Profile [14]. Two videocameras were used to record all tasks and interactions.

\section{AAC book-reading interaction}

The stimuli for both interactions were developed using Boardmaker with Speaking Dynamically Pro ${ }^{\circledR}$. Two books were used in this study, Brown Bear, Brown Bear, What Do You See (1995) and Polar Bear, Polar Bear, What Do You Hear (1991) $[15,16]$. The pages were scanned onto a computer and then displayed on a touchscreen tablet PC with a 12.1 inch viewable screen and resolution set to $1,280 \times 800$. The PC pages were arranged the same as in each book, with the addition of three active buttons on each screen. Pressing on the animal activated the programmed animal sound and a red outline appeared around the animal. The actual books were also present in each experimental interaction. Each child participated in two book/AAC interactions, aligned and divided. In the aligned interaction, the AAC system was placed at the chest level of the experimenter directly below her face with the book to the side. In the divided interaction the system was placed to one side of the experimenter and the physical book to the other side. The aligned interaction was always presented first since there was a suggested benefit from this interaction, previous research revealed no order effect and since it was uncertain the extent to which the children would participate in the tasks [4].

During the AAC interaction the child was allowed to sit on the floor in front of the caregiver or on the partner's lap. Regardless the position of the experimenter, system and book were identical across participants. The experimenter presented all pages of the book on both the system and the physical book. The researcher read the page and then waited five seconds. If the participant did not access the animal sound, the researcher would model by pressing the button on the system and eliciting the sound. This entire book reading process was then repeated with the second book and the system in the aligned position after a short break. There mean duration of the interactions was 233 seconds for the aligned interaction and 216 for the divided interaction.

\section{Joint attention skills}

The abridged version of the Early Social Communication Scale was administered one to two days after the AAC interactions [13]. The ESCS includes 25 semi structured items that measure social communication behaviors such as initiating and responding to joint attention (IJA and RJA) and initiating and responding to behavioral requests (IBR and RBR). The assessment takes between 15 and 25 minutes depending on the child's behavior and responses. See detailed administration procedures of the ESCS [13].

\section{Caregiver-child interaction}

Caregivers were instructed to play with their children as they would at home for ten minutes. Toys included stackable cups, a train set, a ball, a puppet, a book, connecting blocks, cars, an airplane, Little People ${ }^{\circledR}$ figures and vehicles, and animal figures.

\section{Attrition}

Participant 7, the youngest participant, withdrew from the first AAC interaction after completing eight pages of the first book. Her data are not described in the results. During the second interaction of Participant 3's session, the AAC system turned off and had to be restarted; the last three pages of the his second interaction were omitted. Participant 4 refused to participate in the caregiver-child interaction.

\section{Coding}

A coding scheme based on Adamson and Bakeman (1984) was applied to both the AAC book reading interactions and the caregiver-child interactions [17]. Passive JA (PJA) was coded when the child and experimenter (or caregiver) attended to the same object (either the book or the system) for a minimum of three seconds. This included momentary glances at the experimenter or caregiver, but there was little evidence that the child was truly engaged with or aware of the attentional focus of the experimenter (or caregiver). Coordinated JA (CJA) was coded when the experimenter (or caregiver) and child attended to the same object and the child actively and repeatedly acknowledged the experimenter's (or caregiver's) participation in the interaction for at least three seconds. Evidence of the child's awareness of the experi- 
menter (or caregiver) engaging with him or her included triadic gaze shifts and/or verbalizations about the ongoing activity or object. An additional code (Aided Symbol Infused Joint Attention) was adapted for the AAC interactions only [4]. This code was defined as an episode involving three shifts among both the book and the system (i.e. engagement shifts from the book to the system and then back to the book). The child could not linger on either the book or system longer than three seconds, and the overall episode had to last a minimum of three seconds.

An independent coder blind to the goals of the study was trained by the second author on the coding scheme. Reliability analyses were conducted on a second by second basis. An agreement was counted only if a given second was coded as the same state by both individuals. Reliability scores for $28 \%$ of the AAC interaction and the caregiver-child interactions yielded Cohen's Kappas of 0.81 (94.2\% agreement) and .88 (91.9\% agreement), respectively; both Kappas indicated a strong level of agreement. Interrater reliability for the ESCS measure was $94.6 \%$.

\section{RESULTS}

\section{Patterns of engagement during AAC interactions by social communication group}

Based on closer inspection of the communication and JA engagement states during the AAC interactions, two subgroups emerged: beginning coordinators and active coordinators. In the aligned AAC condition four of the seven participants engaged in Passive Joint Attention (PJA) and Augmented Coordinated Joint Attention (ASIJA) but no Coordinated Joint Attention (CJA); and in the divided condition, they engaged only in PJA. In the parent-child free play interaction they generally engaged in more PJA than CJA. These four participants will be discussed first as a group and then individually on a more qualitative basis. The other three children (participants 5-7) showed larger variability in their results and will be discussed individually. Caregiver report of language skills (see Table 2) and performance on the ESCS (see Table 2) supported the subgroups based on the AAC interactions (see Figures 1A, B, and 2).

\section{Beginning coordinators}

In the aligned interaction all of the participants in this group (participants 1-4) engaged in PJA but they did not engage in CJA. These children engaged in a noteworthy amount of Aided Symbol- Infused Joint Attention (ASIJA) in this interaction. As shown in Figure 1A, episodes of PJA in this group ranged from 8 to 11 and episodes of ASIJA ranged from 3 to 6 . A similar pattern of findings emerged for the duration of time spent in these engagement states; PJA ranged from 107-173 seconds and ASIJA ranged from 16-50 seconds.

Participant 1 frequently looked back and forth between the book and the system when the experimenter turned the pages. He used very little language during the interaction; his vocalizations and utterances were characterized by grunting, squealing, vowel production and one single word approximation ('book'). When the stimuli changed, he became more engaged and more interested in looking at both the system and the book. He activated the button on the display 13 times with his finger to activate the animal sound. When the system was activated, participant 1 produced vowel like vocalizations and squeals in response to the sounds.

In the divided interaction, participants 1-4 engaged only in PJA as shown in Figure 1B. Frequency of episodes ranged from 2 to 4 . Two participants ( 1 and 2) engaged in few episodes of PJA with either the book or the AAC system which were high in duration. The duration of PJA for participants 1

Table 2. Select Early Social Communication Scale (ESCS) Scores

\begin{tabular}{lccccccccccc}
\hline Part & Low IJA & High IJA & Low RJA & High RJA & Low IBR & High IBR & RBR Passes & RBR Fails & ISI & RSI \\
\hline 1 & 2 & 0 & $X$ & $X$ & 6 & 1 & 67 & 33 & 0 & 0 \\
2 & 13 & 3 & 100 & 75 & 12 & 3 & 64 & 36 & 7 & 11 \\
3 & 0 & 0 & 0 & $X$ & 4 & 0 & 6.7 & 93.3 & 0 & 0 \\
4 & 1 & 0 & 60 & 0 & 2 & 0 & 40 & 60 & 0 & 4 \\
5 & 7 & 1 & 100 & 100 & 6 & 7 & 100 & 0 & 0 & 7 \\
6 & 19 & 6 & 60 & 50 & 10 & 5 & 53.3 & 46.7 & 11 & 18 \\
7 & 4 & 0 & 33 & $X$ & 6 & 9 & 46.7 & 53.3 & 4 & 2 \\
\hline
\end{tabular}

IJA = Initiating Joint Attention; RJA=Responding to Joint Attention; IBR=Initiating Behavioral Response; RBR=Responding to Behavioral Request; $|S|=$ Initiating Social Interaction; RSI = Responding to Social Interaction; $X=$ the child did not complete the task(s) associated with this measure. 

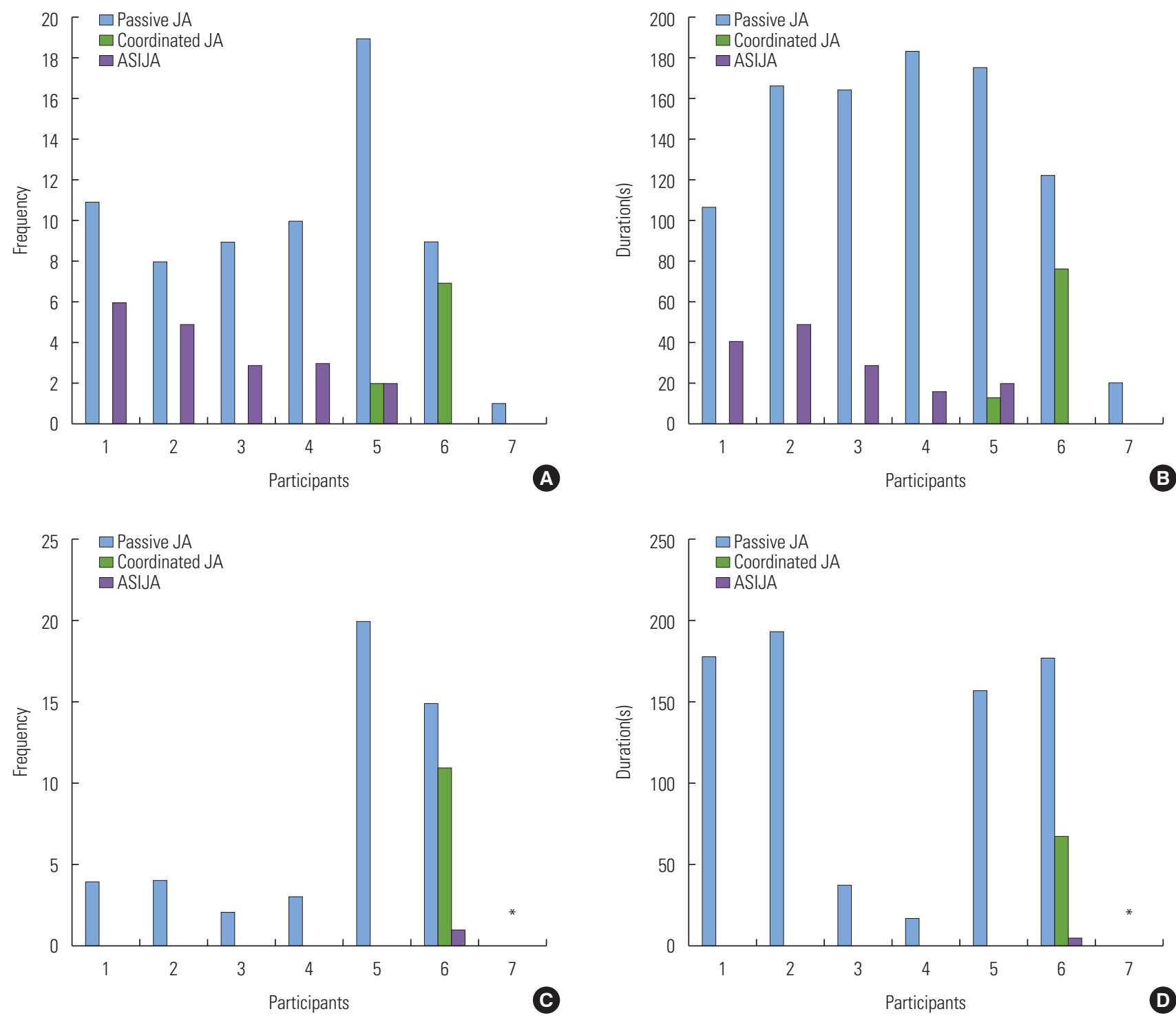

Figure 1. (A, B) The frequency and duration of joint attention states in the aligned book-reading condition. Beginning communicators are Participants 1-4 and Active Communicators are Participants 5-7. (C, D) The frequency and duration of joint attention states in the divided book-reading condition. Beginning communicators are Participants 1-4 and Active Communicators are Participants 5-7.

and 2 were 178 and 194 seconds, respectively. In comparison, the other two Beginning Coordinators (participants 3 and 4) displayed few, short episodes of PJA lasting for 38 and 17 seconds respectively. Participant 1 engaged in three extended episodes of PJA. During these episodes, he kneeled or laid next to the AAC system with his face very close and listened to the animal noises as he activated the system. Once again he used very little language or vocalizations and he spent a large amount of the interaction time engaged only with the system. His participation during this interaction stands in contrast to the episodes of attention shifting he displayed between the device and the book during the aligned interaction.

\section{Active coordinators}

The Active Coordinators group (Participants 5 and 6) was based on their patterns of CJA engagement during the AAC interactions. These participants both engaged in CJA during the aligned AAC interaction. There were 2 episodes (Participant 5) and 7 episodes (Participant 6) of CJA and 9 episodes (Participant 5) and 19 episodes (Participant 6) of PJA. Participant 5 engaged in 2 episodes (duration $=19$ seconds) of ASIJA, but Participant 6 did not engage in any ASIJA during this interaction. Similar patterns emerged for the duration of CJA (Participant 5: 12 seconds; Participant 6: 76 seconds) and PJA (Participant 5: 176; Participant 6: 123 seconds). 

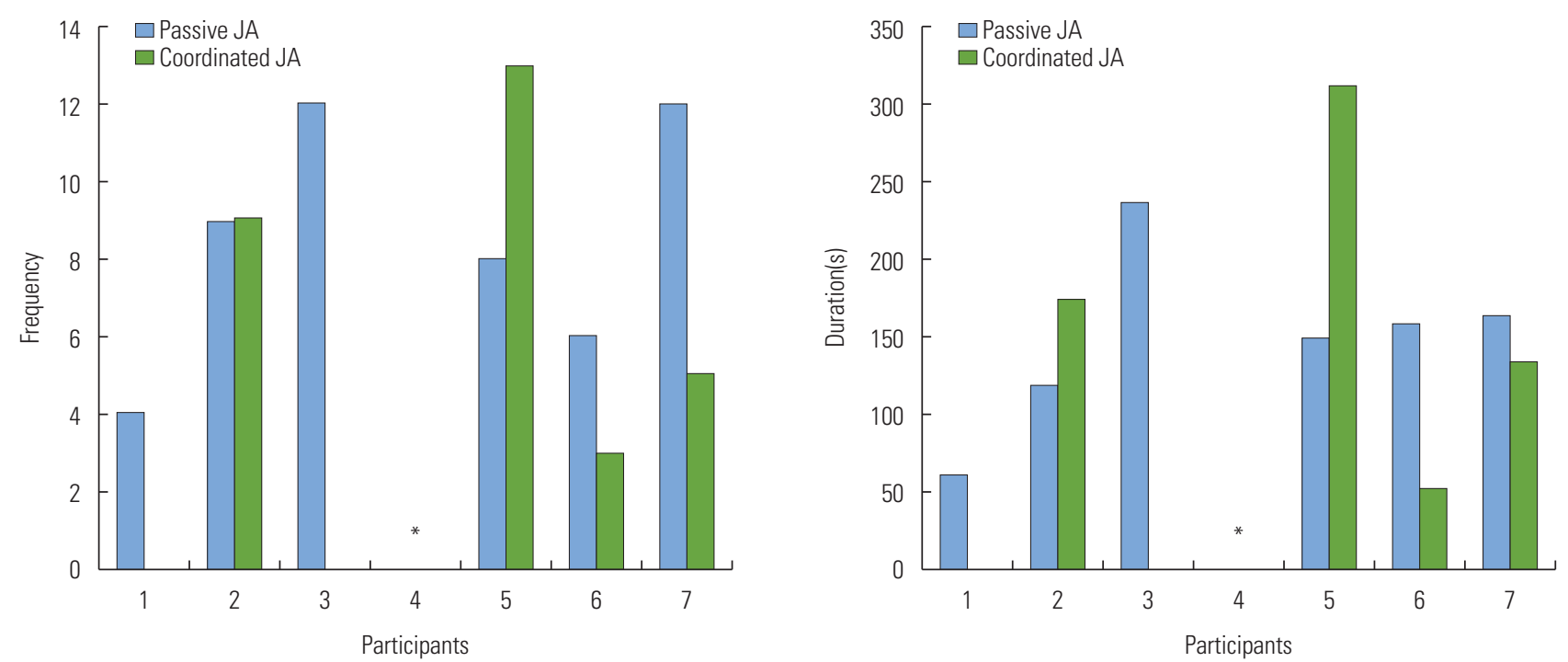

Figure 2. The frequency and duration of joint attention states in the caregiver-child free play interaction. Beginning communicators are Participants 1-3 and Active Communicators are Participants 5-7. Participant 4 did not complete the task.

During the aligned interaction, participant 5 predominantly engaged in frequent, short episodes of PJA. He did, however, shift his gaze between the AAC system and the experimenter several times. He requested help to activate the system by raising his hand and verbally approximated 'help' in the beginning of the interaction. He also shifted his attention between the device and the book twice. In contrast, during the divided AAC interaction, participant 5 engaged only in PJA (duration $=157$ seconds). Though he shifted his attention from the book to the system and vice versa, he lingered on either the book or the system for longer than 3 seconds; thus, these episodes were not coded as ASIJA. His frequent switching (often involving turning 180 degrees from book to system) implied that he understood the correspondence between book and system, but the placement of the system may not have been conducive to facilitating ASIJA. Participant 6 engaged in all three JA states as displayed in Figure 2. Duration findings displayed similar patterns (PJA: 178 seconds; CJA: 68 seconds; ASIJA: 5 seconds). She frequently commented on new pages and animals in the book to the experimenter. She would repeat lines from the story (e.g. 'green horse, green horse') and talk to the animals on the page (e.g. 'hi fish!').

\section{Patterns of engagement during caregiver-child interactions and ESCS results by group beginning coordinators}

Engagement in PJA episodes for Participants 1-3 ranged from 4 to 12 and CJA episodes ranged from 0 to 9 . Duration rates revealed similar patterns; PJA ranged from 62 to 239 seconds,
CJA ranged from 0-173 seconds. Some differences were noted within this group. Participants 1 and 3 engaged only in episodes of PJA. That is, they did not coordinate their attention with their caregivers at any time during the caregiver-child interaction. In contrast, Participant 2 exhibited an equal number of episodes of PJA and CJA (nine of each). ESCS results for the beginning coordinators support the JA engagement patterns in the caregiver-child interaction (See Table 2). Participants 1 and 3 scored low for both high and low IJA and RJA behaviors. Participant 1 engaged in a shorter free play session (3 minutes and 22 seconds) because he became agitated and withdrew. He engaged in one episode of PJA with his mother that lasted a total of 62 seconds. In contrast, Participant 2, who engaged approximately $29.9 \%$ of time in CJA with his father, also scored highly in all joint attention behaviors during the ESCS. It is important to note that the father of participant 2 was extremely supportive of coordinating attention during play with his child. He followed into his son's focus of attention as opposed to directing his child's attention elsewhere. In the context of JA episodes, he talked about the toys his son was engaged with and he frequently labeled objects his son picked up (e.g., 'that's an airplane!').

\section{Active coordinators}

During the caregiver-child interaction, there were 13 (participant 5) and 3 (participant 6) episodes of CJA. There were 8 (participant 5) and 6 (participant 6) episodes of PJA. PJA ranged from Similar trends emerged for duration of CJA (par- 
ticipant 5: 313 seconds; participant 6: 50 seconds) and PJA (participant 5: 150 seconds; participant 6: 158 seconds). This pattern is reflective of their performance during the AAC interactions in that both of these participants frequently shared focus as well as coordinated their attention with others. The results from the ESCS show patterns similar to those in the caregiver-child interaction for the active coordinator group. Both participants 5 and 6 received high scores in both IJA and RJA behaviors (see Table 2). These children were both frequently initiating joint attention with the experimenter during testing. Although participant 2 also had high IJA and RJA scores, he did not demonstrate CJA in any condition.

\section{DISCUSSION}

Across both interactions, participants exhibited more passive than coordinated joint attention. Findings suggest communication and play profiles, including ESCS results, caregiver report of language and free play interactions may be effective in contextualizing the patterns of engagement during structured tasks. It is important to note that the findings do not just reflect age related change as observed in other studies of JA engagement with AAC systems with beginning communicators who are typically developing [4]. Rather, in the present study, the patterns of JA engagement observed in were attributable to the social communication and language status of the children, not maturation.

In the aligned interaction, children in both social communication groups were more likely to shift and coordinate their attention between the system and the experimenter or the book (ASIJA). The divided interaction, in contrast, led to greater frequency of PJA and disengagement. One explanation may be that it was less demanding to shift attention when the object and experimenter were physically closer together (i.e. in the aligned interaction). Fewer social and cognitive resources were demanded of the child, thereby resulting in greater frequency and duration of engagement and exchange. Children in the divided interaction may have monitored the experimenter without receiving a CJA code, which allowed for occasional, quick glances without considering them as coordinating attention. JA around an object may not actually require gaze alternation [18]. Shorter gaze requirements and/or the role of peripheral gaze may need to be considered based on the tendencies of children with ASD to engage in minimal eye contact. It is also possible that the divided AAC interaction led to overarousal and/or gaze aversion as means of calming themselves and reducing the cognitive demands of the situation $[19,18]$. When children with ASD do not make eye contact with communication partners, they may be attempting to reduce the demands in difficult communicative situations. While this may be an issue in both of the AAC interactions, the divided interaction placed more demand on participants.

\section{Beginning and active coordinators}

Based on children's engagement during the two interaction conditions, two groups emerged. Children in the Beginning Coordinators group frequently shifted attention between the book and system (ASIJA) in the aligned interaction while this pattern was not observed in children without disabilities [4]. This action may be a bridge between PJA and CJA. In ASIJA, children have acquired the skill of shifting and dividing one's attention, but not yet included the additional demand of social interaction. If children then master the social aspect of a communicative interaction, they may eventually be able to integrate these skills to develop CJA abilities (i.e. shift their attention between the experimenter and the system). While this is not the developmental trajectory of children without disabilities, the timeline for developing these skills in children with ASD may be protracted due to high level social and cognitive demands. In contrast, children in the Active Coordinators group engaged in more episodes of CJA, particularly in the aligned condition. They used language to support their engagement and they were more likely to initiate actions with the AAC system. Relative to the Beginning Coordinator counterparts, they did not engage in as many episodes of ASIJA because their shifts in attention were shared with the experimenter and the system as opposed shifting attention between the system and the book.

\section{Limitations}

Due to the preliminary nature of this work, a smaller sample size was pursued. Though we were not able to detect significant differences across the conditions, the interactions engineered afforded us the opportunity to examine children's response to the conditions as a function of his or her communication level.

\section{Clinical implications}

Children operating at the beginning coordinator level clearly need more assistance in sharing and shifting their attention. Coordinating attention is challenging; although, it is preferable that the child shift attention away from the device after se- 
lecting a message to communicate. Since ASIJA may be a bridge towards CJA, therapy for individuals using aided AAC should target ASIJA behaviors and scaffold social interaction onto this framework. Knowing the difficulties with shifting and dividing attention for children with ASD and the caveats associated with AAC use, intervention expectations should be managed accordingly.

The recommendations for active coordinators are different. Because they have skills in coordinating attention among themselves, their social communication partners, the AAC system, and objects, their goals would be more centered on increasing their initiation with the system. Building in prompts into the AAC system (e.g., questions, directions) may be one direction to pursue. Other intervention models suggest embedding JA interactions into pretend play contexts [3]. Therapy approaches which focus on combining social interaction with direct training will lead to increases in skills of initiating and maintaining JA with others for children with ASD [20].

\section{CONFLICT OF INTEREST}

The author has no conflict of interests.

\section{ACKNOWLEDGMENTS}

The authors would like to thank Madeline Wilson and Allie Sidwell for their assistance with data collection, transcription, and coding. This project was partially funded by Ohio University Institutional start-up funds awarded to the third author. Please note that the authors of this manuscript do not have a direct financial relation with the commercial identities (Speaking Dynamically Pro ${ }^{\circledR}$ software \& Little People ${ }^{\circledR}$ ) mentioned in this work.

\section{REFERENCES}

1. Kasari C, Kaiser A, Goods K, Nietfeld J, Mathy P, Landa R, Murphy S, Almirall D. Communication interventions for minimally verbal children with autism: sequential multiple assignment randomized trial. Journal of the American Academy of Child and Adolescent Psychiatry. 2014;53(6):635-646.

2. Adamson L, Bakeman R, Deckner D, Romski M. Joint engagement and the emergence of language in children with autism and down syndrome. Journal of Autism \& Developmental Disorders. 2009;39(1):84-96.

3. Kasari C, Freeman S, Paparella T. Joint attention and symbolic play in young children with autism: a randomized controlled in- tervention study. Journal of Child Psychology and Psychiatry 2005;48(5):523.

4. Smith V, Mirenda P, Zaidman-Zait A. Predictors of expressive vocabulary growth in children with autism. Journal of Speech, Language \& Hearing Research. 2007;50(1):149-160.

5. Wetherby A. Prizant B. Autism spectrum disorders: A transactional developmental perspective. Baltimore, MD: Paul H. Brookes Publishing Co; 2000.

6. Mirenda P. Toward functional augmentative and alternative communication for students with autism: manual signs, graphic symbols, and voice output communication aids. Language, Speech, and Hearing Services in Schools. 2003;34: 203-216.

7. Beukelman D. Mirenda P. Augmentative and alternative communication; supporting children and adults with complex communication needs. Baltimore, Maryland: Paul H. Brooks Publishing; 2005.

8. Schlosser R, Wendt O. Effects of augmentative and alternative communication intervention on speech production in children with autism: A systematic review. American Journal of Speech Language Pathology. 2008;17:212-230.

9. Benigno JP, McCarthy JW. Aided symbol-infused joint engagement. Child Development Perspectives. 2012;6:181-186.

10. Spencer P, Bodner-Johnson B, Gutfreund M. Interacting with infants with a hearing loss: What can we learn from mothers who are deaf? Journal of Early Intervention. 1992;16:64-78.

11. Shire S, Jones N. Communication partners supporting children with complex communication needs who use AAC. Communication Disorders Quarterly. 2015;37:3-15.

12. Smith J, McCarthy JW, Benigno JP. The effect of high-tech AAC system position on the joint attention of infants without disabilities. Augmentative and Alternative Communication. 2009;25(3):165175.

13. Mundy P, Delgado C, Block J, Venezia M, Hogan A, Seibert J. A manual for the abridged early social communication scales (ESCS) [Internet]. University of California Davis MIND Institute [cited 2009 April 8]. Available from: http://ucdmc.ucdavis.edu/ mindinstitute/ourteam/faculty/mundy.html.

14 Wetherby A, Prizant B. Communication and symbolic behavior scales developmental profile. Baltimore, MD: Paul H. Brookes Publishing Co; 2002.

15. Martin B. Brown bear, brown bear, what do you see? New York: Henry Holt and Company; 1995.

16. Martin B. Polar bear, polar bear, what do you hear? New York: Henry Holt and Company; 1991.

17. Bakeman R, Adamson L. Coordinating attention to people and objects in mother- infant and peer infant interactions. Child Development. 1984;55(4):1278-1289.

18. Akhtar N, Gernsbacher MA. On privileging the role of gaze in infant social cognition. Child Development Perspectives. 2008;2(2): 59-65.

19. Phelps F, Doherty-Sneddon G, Warnock H. Helping children think: Gaze aversion and teaching. British Journal of Developmental Psychology. 2006;24:577-588.

20. Therrien M, Light J, Pope L. Systematic review of the effects of interventions to promote peer interactions for children who use aided AAC. Augmentative and Alternative Communication. 2016;32: 81-93. 